\title{
Trend to equilibrium for discrete coagulation equations with strong fragmentation and without balance condition
}

\author{
Nicolas FournieR ${ }^{1}$ and Stéphane MischleR ${ }^{2}$
}

\begin{abstract}
The coagulation-fragmentation equation describes the concentration $f_{i}(t)$ of particles of size $i \in \mathbb{N} /\{0\}$ at time $t \geq 0$, in a spatially homogeneous infinite system of particles subjected to coalescence and break-up. We show that when the rate of fragmentation is sufficiently stronger than that of coalescence, $\left(f_{i}(t)\right)_{i \in \mathbb{N} /\{0\}}$ tends to an unique equilibrium as $t$ tends to infinity. Although we suppose that the initial datum is sufficiently small, we do not assume a detailed balance (or reversibility) condition. The rate of convergence we obtain is exponential.
\end{abstract}

Key words : Coalescence, Fragmentation, Differential equations, Equilibrium.

\section{Introduction and result}

Consider an infinite system of particles characterised by their size $i \in \mathbb{N}^{*}:=\mathbb{N} /\{0\}$. Assume that two particles of size $i \in \mathbb{N}^{*}$ and $j \in \mathbb{N}^{*}$ coalesce at rate $a_{i, j}$ to give a particle of size $i+j$. Suppose also that each particle of size $i+j$ breaks up to give two particles of size $i \in \mathbb{N}^{*}$ and $j \in \mathbb{N}^{*}$ at rate $b_{i, j}$. We will assume in the whole paper that for all $i, j$ in $\mathbb{N}^{*}$,

$$
0 \leq a_{i, j}=a_{j, i} ; \quad 0 \leq b_{i, j}=b_{j, i} .
$$

Denote by $f_{i}(t)$ the concentration (i.e. number per unit of volume) of particles of size $i \in \mathbb{N}^{*}$ at time $t \geq 0$. Then $f=\left(f_{i}(t)\right)_{i \in \mathbb{N}^{*}, t \geq 0}$ satisfies the coagulation-fragmentation equations $[7,2]$ :

$$
\forall i \in \mathbb{N}^{*}, \quad \frac{d}{d t} f_{i}(t)=Q_{i}(f(t)), t \in(0, \infty) ; \quad f_{i}(0)=f_{i}^{i n},
$$

where the initial datum $\left(f_{i}^{i n}\right)_{i \in \mathbb{N}^{*}}$ is given. The coagulation-fragmentation operator is defined, for $c=\left(c_{i}\right)_{i \in \mathbb{N}^{*}}$ a sequence of nonnegative numbers and $i \in \mathbb{N}^{*}$, by

$$
Q_{i}(c):=\frac{1}{2} \sum_{j=1}^{i-1}\left(a_{j, i-j} c_{j} c_{i-j}-b_{j, i-j} c_{i}\right)-\sum_{j=1}^{\infty}\left(a_{i, j} c_{i} c_{j}-b_{i, j} c_{i+j}\right) .
$$

These equations have the following physical meaning: particles of size $i$ appear due to coalescence of smaller particles at rate $\frac{1}{2} \sum_{j=1}^{i-1} a_{j, i-j} f_{j} f_{i-j}$, the factor $1 / 2$ avoiding to count twice each pair of particles. Particles of size $i$ also appear by break-up of greater particles at rate $\sum_{j=1}^{\infty} b_{i, j} f_{i+j}$. Disappearance of particles of size $i$ occur by coalescence at rate $f_{i} \sum_{j=1}^{\infty} a_{i, j} f_{j}$ and by fragmentation at rate $\frac{1}{2} f_{i} \sum_{j=1}^{i-1} b_{j, i-j}$.

One of the main problems is the long time behavior of solutions to (1.2). On the one hand, it is proved in [3] that, in the long time asymptotic:

\footnotetext{
${ }^{1}$ Institut Elie Cartan, Campus Scientifique, BP 239, 54506 Vandoeuvre-lès-Nancy Cedex, France, fournier@iecn.unancy.fr

${ }^{2}$ Ceremade - UMR 7534, Université de Paris IX-Dauphine, Place du Maréchal De Lattre de Tassigny, 75775 Paris Cedex 16, France, mischler@ceremade.dauphine.fr
} 
(i) when $a \equiv 0$ and $b>0$ (pure fragmentation case) all particles break into monomers, that is $f_{i}(t) \rightarrow 0$ when $t \rightarrow \infty$ for any $i \geq 2$;

(ii) when $b \equiv 0$ and $a>0$ (pure coagulation case) each particle's mass increases to infinity, that is $f_{i}(t) \rightarrow 0$ when $t \rightarrow \infty$ for any $i \geq 1$.

On the other hand, one may assume a detailed balance condition: there exists a nonnegative sequence $\mathcal{M}=\left(\mathcal{M}_{i}\right)_{i \in \mathbb{N}^{*}}$ such that $\mathcal{M} \not \equiv 0, \sum_{i=1}^{\infty} i \mathcal{M}_{i}<\infty$, and for all $i, j$ in $\mathbb{N}^{*}$,

$$
a_{i, j} \mathcal{M}_{i} \mathcal{M}_{j}=b_{i, j} \mathcal{M}_{i+j}
$$

This structure condition about the rates $a$ and $b$ ensures reversibility properties. This allows one to use entropy methods, see $[1,4,5,11]$, and to show that any solution $f$ to the coagulationfragmentation equation (1.2) converges to an equilibrium.

Let emphasize, that [8] deals with existence of equilibria in a particular case where (1.4) does not hold. To our knowledge, other results concerning trend to equilibrium of solutions to (1.2) were obtained assuming one of the three above conditions.

Our aim in the present paper is to show that trend to equilibrium may hold without any structure condition. We will consider a case where coalescence is weaker than fragmentation, and where the initial datum is small enough. More precisely, we will suppose that we are in a case of strong fragmentation. We assume that there exist some constants $a_{0} \geq 0, b_{0}>0, b_{1}>0, \alpha, \gamma$ and $s$ such that for all $i, j$ in $\mathbb{N}^{*}$,

$$
\begin{gathered}
a_{i, j} \leq a_{0}(i j)^{\alpha}, \quad b_{i, j} \geq b_{0}(i+j)^{\gamma}, \quad \alpha \in[0,1], \quad \gamma \in(-1, \infty), \quad 2+\gamma>2 \alpha, \\
b_{i, j} \leq b_{1}(i+j)^{s} \quad s \in(-1, \infty) .
\end{gathered}
$$

Let us now define the notion of solutions and equilibria we will consider. For $\mu \geq 1$, we set

$$
\ell_{\mu}^{1}:=\left\{\left(c_{i}\right)_{i \in \mathbb{N}^{*}} \in[0, \infty)^{\mathbb{N}^{*}} ; \sum_{i=1}^{\infty} i^{\mu} c_{i}<\infty\right\} .
$$

Definition 1.1 Assume (1.1). A sequence of nonnegative $C^{1}((0, \infty))$ functions $f=\left(f_{i}(t)\right)_{i \in \mathbb{N}^{*}, t \geq 0}$ is an admissible solution to (1.2) associated to an initial datum $f^{i n}=\left(f_{i}^{i n}\right)_{i \in \mathbb{N}^{*}}$ if:

(i) for all $i \in \mathbb{N}^{*}$, (1.2) holds,

(ii) for all $\mu \geq 1, f$ belongs to $C\left([0, \infty), \ell_{1}^{1}\right) \cap C^{1}\left((0, \infty), \ell_{\mu}^{1}\right)$,

(iii) conservation of mass holds, that is for all $t \geq 0, \sum_{i=1}^{\infty} i f_{i}(t)=\sum_{i=1}^{\infty} i f_{i}^{i n}$.

A nonnegative sequence $\left(c_{i}\right)_{i \in \mathbb{N}^{*}}$ is an admissible equilibrium for (1.2) if it is a stationary admissible solution to (1.2).

It is well-known that under (1.5) and (1.6) existence and moment regularisation hold: the following result can be found in $[13,3,6,10]$.

Theorem 1.2 Assume (1.1), (1.5) and (1.6). Let $f^{\text {in }}$ satisfy

$$
m_{1}:=\sum_{i=1}^{\infty} i f_{i}^{i n}<\infty
$$

There exists at least one admissible solution $f$ to (1.2) associated to the initial datum $f_{\text {in }}$.

Let us finally state our result. 
Theorem 1.3 Assume (1.1), (1.5) and (1.6). Let $m_{1}>0$ be fixed, and suppose that

$$
\varphi\left(\frac{a_{0} m_{1}}{b_{0}}\right)<1 \quad \text { with } \quad \varphi(\zeta):=2(32 \zeta)^{2+\frac{1+2 \alpha}{2+\gamma-2 \alpha}}+128 \zeta
$$

(i) There exists an unique admissible equilibrium $c$ to (1.2) such that $\sum_{i=1}^{\infty} i c_{i}=m_{1}$.

(ii) There exists $K, \kappa>0$ (explicitly computable and depending only on $\alpha, \gamma, a_{0}, b_{0}, m_{1}$ ) such that for any admissible solution $f$ to (1.2) with initial datum satisfying (1.8),

$$
\forall t \geq 1, \quad \sum_{i=1}^{\infty} i^{2}\left|f_{i}(t)-c_{i}\right| \leq K e^{-\kappa t}
$$

We believe that Theorem 1.3 is not optimal. The condition that $a_{0} m_{1} / b_{0}$ is small is probably a technical assumption. The main interest of this result compared to $[1,4,5,11]$ is that it does not require any structure condition on the rates $a$ and $b$. The bound conditions on $a$ and $b$ under the form (1.5) are made to simplify presentation, but we may use the same method with other type of assumptions, see [6].

Our condition that $2+\gamma>2 \alpha$ is not so stringent since if $2+\gamma<2 \alpha$ it is expected that $\sup _{t \in(0, \infty)} \sum_{i=1}^{\infty} i^{2} f_{i}(t)=\infty$, which contradicts the (strong) stability of the system. For example, occurence of gelation has been shown when $\alpha>1 / 2,2+\gamma<2 \alpha$, see $[12,9,10]$.

The rest of the paper is entirely devoted to the proof of Theorem 1.3. Section 2 contains a contraction result, which is applied in Section 3.

\section{A contraction property}

The aim of this section is to present the main tool of the paper, namely a contraction property for the solutions of the coagulation-fragmentation equation.

Theorem 2.1 Let $m_{1}>0$ be fixed. Assume (1.1), (1.5), (1.6) and (1.9). There exists a time $T^{*}>0$ (explicitly computable in terms of $\alpha, \gamma, a_{0}, b_{0}, m_{1}$ ) such that for any pair of admissible solutions $f$ and $g$ to (1.2) associated with some initial data $f^{\text {in }}$ and $g^{\text {in }}$ both satisfying (1.8) (with the same value for $m_{1}$ ), there holds

$$
\forall t \geq T^{*}, \quad \frac{d}{d t} \sum_{i=1}^{\infty} i^{2}\left|f_{i}(t)-g_{i}(t)\right| \leq-\kappa \sum_{i=1}^{\infty} i^{2}\left|f_{i}(t)-g_{i}(t)\right|
$$

with $\kappa:=\frac{b_{0}}{32}\left(1-\varphi\left(a_{0} m_{1} / b_{0}\right)\right)>0$.

We start with a well-known formulation of solutions.

Lemma 2.2 Assume (1.1), (1.5) and (1.6). Let $f$ be an admissible solution to (1.2) associated to an initial data $f^{\text {in }}$ satisfying (1.8). Then for any $\phi: \mathbb{N}^{*} \mapsto \mathbb{R}$ with at most polynomial growth and any $\beta: \mathbb{R} \mapsto \mathbb{R}$ Lipschitz, for all $t>0$,

$$
\begin{aligned}
\frac{d}{d t} \sum_{i=1}^{\infty} \phi(i) \beta\left(f_{i}(t)\right)= & \frac{1}{2} \sum_{i=1}^{\infty} \sum_{j=1}^{\infty} f_{i}(t) f_{j}(t)[\psi(i+j)-\psi(i)-\psi(j)] a_{i, j} \\
& +\frac{1}{2} \sum_{i=1}^{\infty} f_{i}(t) \sum_{j=1}^{i-1}[\psi(j)+\psi(i-j)-\psi(i)] b_{j, i-j}
\end{aligned}
$$

with $\psi(i)=\phi(i) \beta^{\prime}\left(f_{i}(t)\right)$, and the above series converge absolutely. 
Proof The proof follows from a straightforward computation when $\phi$ has a bounded support. Our assumptions on $f$ (see Definition 1.1 (ii)) and on the rates (see (1.5) and (1.6)) allows us the extension to functions with at most polynomial growth.

The key arguments of our proof are contained in the following lemma.

Lemma 2.3 Assume (1.1), (1.5) and (1.6). Consider two admissible solutions $f$ and $g$ to (1.2), associated to some initial data $f^{i n}$ and $g^{\text {in }}$ both satisfying (1.8) with the same value for $m_{1}$. Then, for all $t>0$,

$$
\frac{d}{d t} \sum_{i=1}^{\infty} i^{2}\left|f_{i}-g_{i}\right| \leq\left(2 a_{0} \sum_{i=1}^{\infty} j^{3}\left(f_{j}+g_{j}\right)-\frac{b_{0}}{16}\right) \sum_{i=1}^{\infty} i^{2}\left|f_{i}-g_{i}\right| .
$$

Proof Applying Lemma 2.2 to $f-g$, choosing $\phi(i)=i^{2}$ and $\beta(x)=|x|$, we obtain, with $\psi(i)=$ $i^{2} \operatorname{sign}\left(f_{i}-g_{i}\right)$, for $t>0$,

$$
\begin{aligned}
\frac{d}{d t} \sum_{i=1}^{\infty} i^{2}\left|f_{i}-g_{i}\right|= & \frac{1}{2} \sum_{i=1}^{\infty} \sum_{j=1}^{\infty} a_{i, j}\left[\left(f_{i}-g_{i}\right) f_{j}+g_{i}\left(f_{j}-g_{j}\right)\right][\psi(i+j)-\psi(i)-\psi(j)] \\
& +\frac{1}{2} \sum_{i=1}^{\infty}\left[f_{i}-g_{i}\right] \sum_{j=1}^{i-1}[\psi(j)+\psi(i-j)-\psi(i)] .
\end{aligned}
$$

Using the signs as often as possible, we deduce that

$$
\begin{aligned}
\frac{d}{d t} \sum_{i=1}^{\infty} i^{2}\left|f_{i}-g_{i}\right| \leq & \frac{1}{2} \sum_{i=1}^{\infty} \sum_{j=1}^{\infty} a_{i, j}\left|f_{i}-g_{i}\right| f_{j}\left[(i+j)^{2}-i^{2}+j^{2}\right] \\
& +\frac{1}{2} \sum_{i=1}^{\infty} \sum_{j=1}^{\infty} a_{i, j} g_{i}\left|f_{j}-g_{j}\right| f_{j}\left[(i+j)^{2}+i^{2}-j^{2}\right] \\
& +\frac{1}{2} \sum_{i=1}^{\infty}\left|f_{i}-g_{i}\right| \sum_{j=1}^{i-1} b_{j, i-j}\left[j^{2}+(i-j)^{2}-i^{2}\right] .
\end{aligned}
$$

Making the substitution $(i, j) \mapsto(j, i)$ in the second term leads to

$$
\begin{aligned}
\frac{d}{d t} \sum_{i=1}^{\infty} i^{2}\left|f_{i}(t)-g_{i}(t)\right| \leq & \sum_{i=1}^{\infty}\left|f_{i}(t)-g_{i}(t)\right| \sum_{j=1}^{\infty}\left[i j+j^{2}\right] a_{i, j}\left(f_{j}(t)+g_{j}(t)\right) \\
& -\sum_{i=1}^{\infty}\left|f_{i}(t)-g_{i}(t)\right| \sum_{j=1}^{i-1} j(i-j) b_{j, i-j}
\end{aligned}
$$

Using (1.5), since $\sum_{j=1}^{i-1} b_{j, i-j} \geq \frac{b_{0}}{6} i^{\gamma+1}\left(i^{2}-1\right), 1+\alpha \leq 3$ and $2+\alpha \leq 3$, we deduce

$$
\frac{d}{d t} \sum_{i=1}^{\infty} i^{2}\left|f_{i}-g_{i}\right| \leq-\frac{b_{0}}{6} \sum_{i=1}^{\infty} i^{\gamma+1}\left(i^{2}-1\right)\left|f_{i}-g_{i}\right|+2 a_{0} \sum_{i=1}^{\infty}\left|f_{i}-g_{i}\right| i^{2} \sum_{j=1}^{\infty} j^{3}\left(f_{j}+g_{j}\right) .
$$


But $f$ and $g$ are mass-conserving. Thus $\sum_{i=1}^{\infty} i f_{i}=\sum_{i=1}^{\infty} i g_{i}=m_{1}$ for each $t$, and we deduce that $\left|f_{1}-g_{1}\right| \leq \sum_{i=2}^{\infty} i\left|f_{i}-g_{i}\right|$. Since $\gamma>-1$

$$
\begin{aligned}
\sum_{i=1}^{\infty} i^{\gamma+1}\left(i^{2}-1\right)\left|f_{i}-g_{i}\right| & \geq \frac{3}{4} \sum_{i=2}^{\infty} i^{2}\left|f_{i}-g_{i}\right| \\
& \geq \frac{3}{4} \sum_{i=1}^{\infty} i^{2}\left|f_{i}-g_{i}\right|-\frac{3}{4} \sum_{i=2}^{\infty} i\left|f_{i}-g_{i}\right| \\
& \geq \frac{3}{4} \sum_{i=1}^{\infty} i^{2}\left|f_{i}-g_{i}\right|-\frac{3}{8} \sum_{i=2}^{\infty} i^{2}\left|f_{i}-g_{i}\right| \\
& \geq \frac{3}{8} \sum_{i=1}^{\infty} i^{2}\left|f_{i}-g_{i}\right| .
\end{aligned}
$$

We finally deduce (2.3), gathering (2.7) and (2.8).

The main idea of the proof of Theorem 2.1 is now clear: we have to show that in (2.3), the negative term dominates the positive term. This fact will be a consequence of the following moment estimate.

Lemma 2.4 Let $m_{1}>0$ be fixed. Assume (1.1), (1.5), (1.6) and (1.9). There exists a time $T^{*}$ (explicitly computable in terms of $\left.\alpha, \gamma, a_{0}, b_{0}, m_{1}\right)$ such that, for any admissible solution $f$ to (1.2) with initial datum $f^{\text {in }}$ satisfying (1.8), there holds

$$
\sup _{t \geq T_{*}} \sum_{i=1}^{\infty} i^{3} f_{i}(t) \leq \frac{b_{0}}{128 a_{0}}\left\{\varphi\left(\frac{a_{0} m_{1}}{b_{0}}\right)+1\right\}
$$

with $\varphi$ defined in (1.9).

Proof We break the proof into three steps.

First Step. Let thus $f$ be an admissible solution to (1.2). First note that

$$
\begin{gathered}
\forall i, j \in \mathbb{N}^{*}, \quad(i j)^{\alpha}\left((i+j)^{3}-i^{3}-j^{3}\right) \leq 3 i j\left(i^{\alpha} j^{1+\alpha}+i^{1+\alpha} j^{\alpha}\right) \leq 6\left(i^{2+2 \alpha} j+i j^{2+2 \alpha}\right), \\
\forall i \in \mathbb{N}^{*}, \quad i^{\gamma} \sum_{j=1}^{i-1}\left(i^{3}-j^{3}-(i-j)^{3}\right)=\frac{i^{\gamma+2}\left(i^{2}-1\right)}{2} \geq \frac{3}{8}\left(i^{\gamma+4}-1\right) .
\end{gathered}
$$

Applying Lemma 2.2 with $\phi(i)=i^{3}, \beta(x)=x$, and using (2.10) and (2.11) leads to

$$
\frac{d}{d t} \sum_{i=1}^{\infty} i^{3} f_{i} \leq 3 a_{0} m_{1} \sum_{i=1}^{\infty} i^{2+2 \alpha} f_{i}-\frac{3 b_{0}}{16} \sum_{i=1}^{\infty}\left(i^{\gamma+4}-1\right) f_{i},
$$

for all $t>0$. Using the Young inequality (recall that $2+2 \alpha<4+\gamma$ )

$$
i^{2+2 \alpha} \leq\left(\frac{1}{\varepsilon}\right)^{\frac{3+\gamma}{2+\gamma-2 \alpha}} i+\varepsilon^{\frac{3+\gamma}{1+2 \alpha}} i^{4+\gamma} \quad \text { with } \quad \varepsilon:=\left(\frac{b_{0}}{32 a_{0} m_{1}}\right)^{\frac{1+2 \alpha}{3+\gamma}},
$$

and introducing the notation $M_{\mu}(t)=M_{\mu}(f(t,))=.\sum_{i=1}^{\infty} i^{\mu} f_{i}(t)$, we get

$$
\frac{d}{d t} M_{3} \leq A-B M_{4+\gamma}
$$

with

$$
A:=3 a_{0} m_{1}^{2}\left(\frac{32 a_{0} m_{1}}{b_{0}}\right)^{\frac{1+2 \alpha}{2+\gamma-2 \alpha}}+\frac{3 b_{0} m_{1}}{8}, \quad B:=\frac{3 b_{0}}{32} .
$$


Second Step. We first establish a bound for $M_{3}(1)$. Using the Hölder inequality $M_{3} \leq m_{1}^{\frac{1+\gamma}{3+\gamma}} M_{4+\gamma}^{\frac{2}{3+\gamma}}$, we deduce from (2.14) the differential inequality

$$
\frac{d}{d t} M_{3} \leq A-B^{\prime} M_{3}^{1+\theta} \quad \text { with } \quad \theta:=\frac{1+\gamma}{2}, B^{\prime}:=\frac{3}{32} b_{0} m_{1}^{-\frac{1+\gamma}{2}} .
$$

On the one hand, if, for some $\tau \in(0,1)$, there holds $M_{3}(\tau) \leq\left(2 A / B^{\prime}\right)^{1 /(1+\theta)}$ then the same holds for any $t \in(\tau, 1)$ since $\left(0,\left(2 A / B^{\prime}\right)^{1 /(1+\theta)}\right)$ is an invariant region for the differential inequality (2.16). On the other hand, if for any $t \in(0,1)$, there holds $M_{3}(t) \geq\left(2 A / B^{\prime}\right)^{1 /(1+\theta)}$, then we deduce from (2.16) that for all $t \in(0,1)$,

$$
\frac{d}{d t} M_{3} \leq A\left(\frac{M_{3}}{\left(2 A / B^{\prime}\right)^{1 /(1+\theta)}}\right)^{1+\theta}-B^{\prime} M_{3}^{1+\theta} \leq-\frac{B^{\prime}}{2} M_{3}^{1+\theta}
$$

A direct computation gives

$$
\forall t \in(0,1], \quad M_{3}(t) \leq\left(\frac{2}{\theta B^{\prime} t}\right)^{1 / \theta}
$$

As a conclusion, in any case,

$$
M_{3}(1) \leq \max \left(\left(2 A / B^{\prime}\right)^{1 /(1+\theta)}, \frac{2}{\theta B^{\prime}}\right) .
$$

Note that the RHS term is an explicit function of $\alpha, \gamma, a_{0}, b_{0}, m_{1}$.

Third Step. Next, just using that $M_{4+\gamma} \geq M_{3}$, we deduce from (2.14) that

$$
\frac{d}{d t} M_{3} \leq A-B M_{3} \quad \text { on } \quad(0, \infty)
$$

which in turns implies

$$
\forall t \geq 1, \quad M_{3}(t) \leq M_{3}(1) e^{-B(t-1)}+\frac{A}{B} .
$$

But a straightforward computation using (1.9) and (2.15) shows that

$$
\frac{64 a_{0}}{b_{0}} \frac{A}{B}=\varphi\left(a_{0} m_{1} / b_{0}\right) .
$$

Using (2.19) and (2.21), the fact that by assumption $\varphi\left(a_{0} m_{1} / b_{0}\right)<1$, we deduce that there exists $T^{*}=T^{*}\left(\alpha, \gamma, a_{0}, b_{0}, m_{1}\right)$ such that

$$
\sup _{t \geq T^{*}} \frac{64 a_{0}}{b_{0}} M_{3}(t) \leq \frac{1}{2} \varphi\left(\frac{a_{0} m_{1}}{b_{0}}\right)+\frac{1}{2},
$$

from which (2.9) follows.

Proof of Theorem 2.1. Gathering (2.3) and (2.9), we get for any $t \geq T^{*}$ (with $T^{*}$ defined in Lemma 2.4)

$$
\begin{aligned}
\frac{d}{d t} \sum_{i=1}^{\infty} i^{2}\left|f_{i}-g_{i}\right| & \leq\left(2 a_{0}\left[M_{3}(f(t))+M_{3}(g(t))\right]-b_{0} / 16\right) \sum_{i=1}^{\infty} i^{2}\left|f_{i}-g_{i}\right| \\
& \leq-\frac{b_{0}}{32}\left(1-\varphi\left(a_{0} m_{1} / b_{0}\right)\right) \sum_{i=1}^{\infty} i^{2}\left|f_{i}-g_{i}\right| .
\end{aligned}
$$

This concludes the proof. 


\section{$3 \quad$ Proof of Theorem 1.3}

We begin with a direct consequence of Theorem 2.1.

Lemma 3.1 Let $m_{1}>0$ be fixed. Assume (1.1), (1.5), (1.6) and (1.9). Any admissible solution $f$ to (1.2) satisfies

$$
\forall i \in \mathbb{N}^{*}, \quad \lim _{t \rightarrow \infty}\left|\frac{d}{d t} f_{i}(t)\right|=0 .
$$

Proof For any $h>0$, we introduce the notation $f_{i}^{h}(t)=f_{i}(t+h)$. Then $f^{h}$ is an admissible solution to (1.2). Using Theorem 2.1 with the pair of solutions $f^{h}$ and $f$, we deduce that for all $t>T_{*}$ and $h>0$,

$$
\sum_{i=1}^{\infty} i^{2}\left|f_{i}(t+h)-f_{i}(t)\right| \leq e^{-\kappa\left(t-T^{*}\right)} \sum_{i=1}^{\infty} i^{2}\left|f_{i}\left(T^{*}+h\right)-f_{i}\left(T^{*}\right)\right| .
$$

Dividing the above inequality by $h>0$ and passing to the limit $h \rightarrow 0$ (what is justified by the fact $f \in C^{1}\left((0, \infty) ; \ell_{2}^{1}\right)$ in Theorem 1.2), we get for $t>T^{*}$,

$$
\sum_{i=1}^{\infty} i^{2}\left|\frac{d}{d t} f_{i}(t)\right| \leq e^{-\kappa\left(t-T^{*}\right)} \sum_{i=1}^{\infty} i^{2}\left|\frac{d}{d t} f_{i}\left(T^{*}\right)\right|,
$$

and (3.1) readily follows.

We now are able to conclude.

Proof of Theorem 1.3. We skip the proof into three parts.

Existence of an admissible equilibrium. We start with an admissible solution $f$ to (1.2) associated to some initial datum $f^{\text {in }}$ satisfying (1.8). We first claim that for all $p \geq 1$,

$$
K_{p}:=\sup _{t \geq 1} \sum_{i=1}^{\infty} i^{p} f_{i}(t)<\infty
$$

This can be shown following the line of Steps 1 and 3 of the proof of Lemma 2.4. Indeed, applying Lemma 2.2 with $\phi(i)=i^{p}$ and $\beta(x)=x$ and using (1.5), one obtains the existence of some constants $C_{p}>0, c_{p}>0$, whose values change from line to line, such that

$$
\begin{aligned}
\frac{d}{d t} \sum_{i=1}^{\infty} i^{p} f_{i} & \leq C_{p} \sum_{i=1}^{\infty} i^{p-1+2 \alpha} f_{i} \sum_{j=1}^{\infty} j f_{j}-c_{p} \sum_{i=1}^{\infty}\left(i^{\gamma+p+1}-1\right) f_{i} \\
& \leq C_{p}-c_{p} \sum_{i=1}^{\infty} i^{p} f_{i} .
\end{aligned}
$$

The second inequality holds since $p-1+2 \alpha<\gamma+p+1$ and $\gamma+p+1>p$. Then (3.4) follows from the fact that $f$ is admissible.

This yields the existence of an increasing sequence $t_{n} \rightarrow \infty$ and of a nonnegative sequence $c=$ $\left(c_{i}\right)_{i \in \mathbb{N}^{*}}$ such that for all $\phi: \mathbb{N}^{*} \mapsto \mathbb{R}$ with at most polynomial growth,

$$
\sum_{i=1}^{\infty} \phi(i) c_{i}=\lim _{n \rightarrow \infty} \sum_{i=1}^{\infty} \phi(i) f_{i}\left(t_{n}\right)
$$

In particular,

$$
c \in \bigcap_{\mu \geq 1} \ell_{\mu}^{1} \text { and } \sum_{i=1}^{\infty} i c_{i}=m_{1} .
$$


It is now straightforward to pass to the limit as $n$ tends to infinity in (1.2) and obtain, thanks to Lemma 3.1,

$$
\forall i \in \mathbb{N}, \quad Q_{i}(c)=\lim _{n \rightarrow \infty} Q_{i}\left(f\left(t_{n}\right)\right)=\lim _{n \rightarrow \infty} \frac{d}{d t} f_{i}\left(t_{n}\right)=0 .
$$

As a conclusion $c$ is an equilibrium as stated in Theorem 1.3.

Uniqueness of an admissible equilibrium. We consider two admissible equilibria $c$ and $d$ to (1.2) with same mass $\sum_{i=1}^{\infty} i c_{i}=\sum_{i=1}^{\infty} i d_{i}=m_{1}$. Then $c$ and $d$ are two (constant) admissible solutions to (1.2), so that we may apply Theorem 2.1 , and get

$$
0=\frac{d}{d t} \sum_{i=1}^{\infty} i^{2}\left|c_{i}-d_{i}\right| \leq-\kappa \sum_{i=1}^{\infty} i^{2}\left|c_{i}-d_{i}\right|
$$

which yields $c \equiv d$.

Convergence to equilibrium. Consider finally an admissible solution $f$ to (1.2) associated to some initial data $f^{i n}$ satisfying (1.8). Consider the unique admissible equilibrium $c$ to (1.2) such that $\sum_{i=1}^{\infty} i c_{i}=m_{1}$. Since $c$ is a admissible solution to (1.2), one may apply Theorem 2.1, and obtain that for $t \geq T^{*}=T^{*}\left(\alpha, \gamma, a_{0}, b_{0}, m_{1}\right)$,

$$
\frac{d}{d t} \sum_{i=1}^{\infty} i^{2}\left|f_{i}-c_{i}\right| \leq-\kappa \sum_{i=1}^{\infty} i^{2}\left|f_{i}-c_{i}\right| .
$$

with $\kappa=\kappa\left(\alpha, \gamma, a_{0}, b_{0}, m_{1}\right)$. Hence, for $t>T^{*}$,

$$
\sum_{i=1}^{\infty} i^{2}\left|f_{i}-c_{i}\right| \leq e^{-\kappa\left(t-T^{*}\right)} \sum_{i=1}^{\infty} i^{2}\left|f_{i}\left(T^{*}\right)-c_{i}\right|
$$

On the other hand, we have shown, in the proof of Lemma 2.4 (see (2.19), (2.21)), that for some constant $C=C\left(\alpha, \gamma, a_{0}, b_{0}, m_{1}\right)$, for all admissible solution $g$ to (1.2) with initial data satisfying (1.8), $\sup _{t \geq 1} \sum_{i=1}^{\infty} i^{3} g_{i}(t) \leq C$. Hence

$$
\sup _{t \geq 1} \sum_{i=1}^{\infty} i^{2}\left|f_{i}(t)-c_{i}\right| \leq 2 C
$$

One finally obtains (1.10) with the constants $\kappa$ and $K=2 C e^{\kappa T^{*}}$, which depend only on $\alpha, \gamma, a_{0}, b_{0}, m_{1}$,

\section{References}

[1] M. Aizenman, T.A. Bak, Convergence to equilibrium in a system of reacting polymers, Comm. Math. Phys. 65 (1979), 203-230.

[2] D.J. Aldous, Deterministic and stochastic models for coalescence (aggregation, coagulation): a review of the mean-field theory for probabilists, Bernoulli 5 (1999), 3-48.

[3] J.M. Ball, J. Carr, The discrete coagulation-fragmentation equations : existence, uniqueness, and density conservation, J. Statist. Phys. 61 (1990), 203-234.

[4] J.M. Ball, J. Carr, O. Penrose, The Becker-Döring cluster equations : basic properties and asymptotic behaviour of solutions, Commun. Math. Phys. 104 (1986), 657-692.

[5] J. Carr, Asymptotic behaviour of solutions to the coagulation-fragmentation equations. I. The strong fragmentation case, Proc. Roy. Soc. Edinburgh Sect. A 121 (1992), 231-244. 
[6] F.P. da Costa, Existence and uniqueness of density conserving solutions to the coagulationfragmentation equations with strong fragmentation, J. Math. Anal. Appl. 192 (1995), 892-914.

[7] R.L. Drake, A general mathematical survey of the coagulation equation, in "Topics in Current Aerosol Research (part 2)," International Reviews in Aerosol Physics and Chemistry, Pergamon Press, Oxford, 1972, pp. 203-376.

[8] P.B. Dubovskiı̌, I.W. Stewart, Trend to equilibrium for the coagulation-fragmentation equation, Math. Methods Appl. Sci. 19 (1996), 761-772.

[9] M. Escobedo, S. Mischler, B. Perthame, Gelation in coagulation and fragmentation models, Comm. Math. Phys. 231 (2002), 157-188.

[10] M. Escobedo, Ph. Laurençot, S. Mischler, B. Perthame, Gelation and mass conservation in coagulation-fragmentation models, preprint.

[11] P.-E. Jabin, B. Niethammer, On the rate of convergence to equilibrium in the Becker-Döring equations, J. Differential Equations 191 (2003), 518-543.

[12] I. Jeon, Existence of gelling solutions for coagulation-fragmentation equations, Comm. Math. Phys. 194 (1998), 541-567.

[13] J.L. Spouge, An existence theorem for the discrete coagulation-fragmentation equations, Math. Proc. Cambridge Philos. Soc. 96 (1984), 351-357. 